\title{
Long-term efficacy and safety of exemestane in the treatment of breast cancer
}

\author{
This article was published in the following Dove Press journal: \\ Patient Preference and Adherence \\ 26 March 2013 \\ Number of times this article has been viewed
}

\author{
GA Walker' \\ $M$ Xenophontos ${ }^{2}$ \\ LC Chen ${ }^{3}$ \\ KL Cheung ${ }^{2}$ \\ 'Clinical Oncology, East Midlands \\ Deanery, ${ }^{2}$ Breast Surgery, School \\ of Graduate Entry Medicine and \\ Health; ${ }^{3}$ Medicine Use, School of \\ Pharmacy, University of Nottingham, \\ Nottingham, UK
}

\begin{abstract}
Exemestane, a steroidal aromatase inhibitor, is licensed for postmenopausal patients with estrogen receptor (ER)-positive breast cancer as second-line therapy in metastatic disease following antiestrogen failure and as part of sequential adjuvant therapy following initial tamoxifen. This study is a systematic literature review, evaluating exemestane in different clinical settings. The Ovid Medline (1948-2012), Embase (1980-2012), and Web of Science (1899-2012) databases were searched. Forty-two relevant articles covering randomized controlled trials were reviewed for efficacy and safety, and three for adherence. With regard to efficacy in metastatic disease, exemestane is superior to megestrol acetate after progression on tamoxifen. There is evidence for noninferiority to fulvestrant (following a prior aromatase inhibitor) and to nonsteroidal aromatase inhibitors in the first-line setting. Combined use with everolimus is shown to be more efficacious than exemestane alone following previous aromatase inhibitor use. In the adjuvant setting, a switch to exemestane after 2-3 years of tamoxifen is superior to 5 years of tamoxifen. Exemestane is noninferior to 5 years of tamoxifen as upfront therapy, and may have a role as an extended adjuvant therapy. Used as neoadjuvant therapy, increased breast conservation is achievable. As chemoprevention, exemestane significantly reduces the incidence of breast cancer in "at-risk" postmenopausal women. Exemestane is associated with myalgias and arthralgias, as well as reduced bone mineral density and increased risk of fracture, which do not appear to persist at follow-up, with subsequent return to pretreatment values. Compared with tamoxifen, there is a reduced incidence of endometrial changes, thromboembolic events, and hot flashes. Limited evidence shows nonadherence in $23 \%-32 \%$ of patients. Evidence is growing in support of exemestane in all clinical settings. It is generally more efficacious and has a better safety profile than tamoxifen. How it compares with the nonsteroidal aromatase inhibitors remains to be established. Further studies are required on adherence to ensure that maximum benefit is obtained.
\end{abstract}

Keywords: breast cancer, exemestane, review, adherence, metastatic, adjuvant

\section{Introduction}

Exemestane is a third-generation, selective, irreversible, steroidal aromatase inhibitor and inhibits the enzyme aromatase in peripheral fat. This action reduces levels of circulating estrogen in postmenopausal women. Unlike tamoxifen, exemestane has no partial estrogen agonist activity. This study reviewed the literature systematically to evaluate the long-term clinical efficacy and safety of exemestane in the treatment of breast cancer and to identify the findings of studies, if any, related to adherence with the drug in clinical practice. An attempt will be made to contrast this compound against the "gold standard" endocrine agent, tamoxifen, as well as the nonsteroidal third-generation aromatase inhibitors. 


\section{Literature search}

A selective review of the literature in the Ovid Medline (1948-2012), Embase (1980-2012), and Web of Science (1899-2012) database using the search terms "exemestane" and "breast cancer" or "breast neoplasm" was undertaken, yielding 352 articles. The search was then limited to: humans; female; randomized controlled trial; invasive carcinoma; post-menopausal; and English language. Studies were excluded if outcomes were nonclinical, involved ductal carcinoma in situ (noninvasive), or pilot studies. The articles were first evaluated by title and abstract, yielding 62 results. After reading the full text, 42 remained (Figure 1).

A separate search was conducted for adherence. The aforementioned databases were searched using the terms "exemestane" and "adherence or nonadherence", "compliance or noncompliance", and "persistence or nonpersistence," finding 173 articles. After reading the title and abstract, 18 articles were identified for review of full text. Of these, three articles were selected by differentiating exemestane from the class of aromatase inhibitors (Figure 2).

\section{Efficacy}

\section{Metastatic disease}

Exemestane was first licensed for use in postmenopausal patients having metastatic breast cancer with estrogen receptor (ER)-positive tumors following progression on first-line antiestrogen therapy (tamoxifen). There are also emerging data to support its use in the first-line metastatic setting. Five key Phase III trials will be discussed in turn. The trials can be assigned to three groups according to the timing of intervention with exemestane (Table 1).

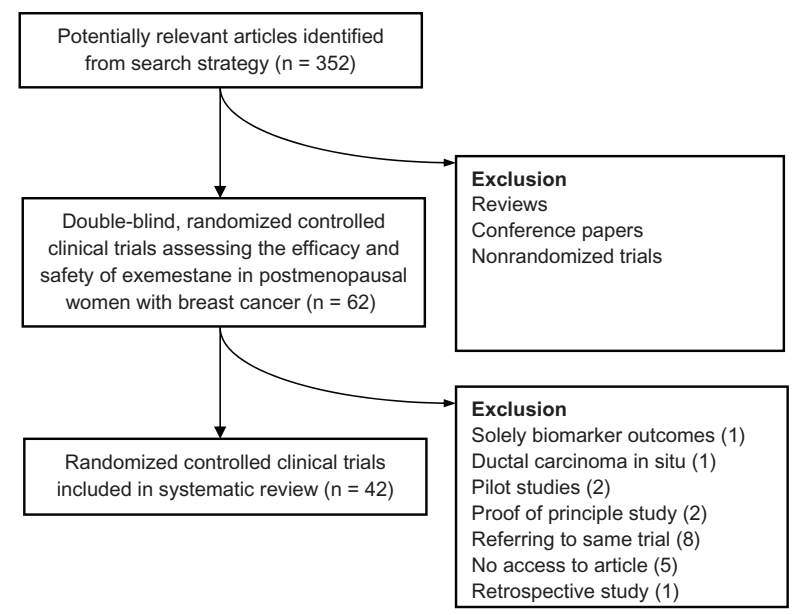

Figure I Process of identifying eligible studies addressing efficacy and safety of exemestane in the systematic review.

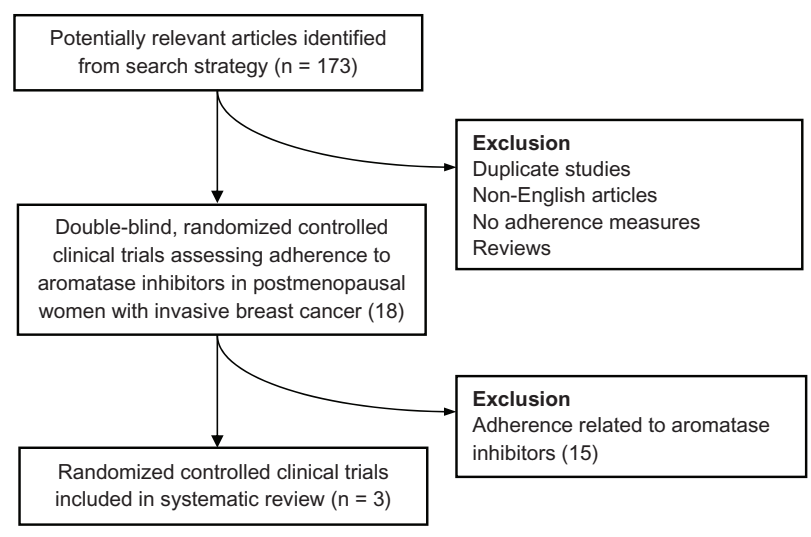

Figure 2 Process of identifying eligible studies addressing adherence with exemestane in the systematic review.

\section{Following progression on tamoxifen}

The Exemestane Study Group performed a trial assessing the efficacy of exemestane versus megestrol acetate $(n=769)$, following progression of metastatic breast cancer on tamoxifen. ${ }^{1}$ The results demonstrated improved response rates (15\% versus $12.4 \%)$ and better progression-free survival (20.3 versus 16.6 weeks, $P=0.037$ ) with exemestane. At a median follow-up of 49 weeks, median overall survival was not reached in the exemestane arm, whilst it was reported as 123 weeks in the megestrol acetate $\operatorname{arm}(P=0.039)$. The recommendation of the group was that exemestane should be considered more efficacious than megestrol acetate in this setting.

A Phase III GINECO (Groupe d'Investigateurs Nationaux pour L'Etude des Cancers Ovarien) trial identified in our search assessed exemestane versus exemestane and celecoxib in the setting of tamoxifen failure. ${ }^{2}$ Celecoxib has been reported to have antitumor effects and synergistic properties when combined with an aromatase inhibitor, and has been well tolerated in Phase II studies. ${ }^{3}$ On analysis of the 157 patients recruited, there was a trend towards improved progressionfree survival in the combination arm of 9.6 versus 5.1 months. This identified a drug combination with potentially increased efficacy compared with monotherapy. However, following on from the increased cardiovascular events with rofecoxib in the APPROVe (Adenomatous Polyp PRevention On Vioxx) study, this trial was terminated before reaching accrual. ${ }^{4}$

\section{Following progression on nonsteroidal aromatase inhibitors}

EFECT (Evaluation of Faslodex versus Exemestane Clinical Trial) assessed the efficacy of fulvestrant, a pure estrogen antagonist (intramuscular injection every 28 days) and exemestane in 693 patients with metastatic breast 
Table I A summary of the trials assessing the efficacy of exemestane in the metastatic, adjuvant, neoadjuvant and chemoprevention setting

$\begin{array}{llll}\text { Trial Drug in arm I } & \text { Drug in arm } 2 & \text { Ratio (95\% CI) }\end{array}$

Value

\section{Metastatic disease}

Kauffmann et al' Exemestane study group trial

\begin{tabular}{|c|c|}
\hline $\begin{array}{l}\text { Exemestane } \\
(n=366)\end{array}$ & $\begin{array}{l}\text { Megestrol Acetate } \\
(n=403)\end{array}$ \\
\hline $15.0(11.5-19.1)$ & $12.4(9.4-16.0)$ \\
\hline $20.3(16.1-24.7)$ & $16.6(15.6-22.9)$ \\
\hline $\begin{array}{l}\text { Exemestane } \\
(n=342)\end{array}$ & Fulvestrant $(n=35 \mathrm{I})$ \\
\hline $6.7(n=270)$ & $7.4(n=270)$ \\
\hline $31.5(n=270)$ & $32.2(n=270)$ \\
\hline 3.7 & 3.7 \\
\hline 9.8 & 13.5 \\
\hline 5.5 & 7.5 \\
\hline $\begin{array}{l}\text { Exemestane } \\
(\mathrm{n}=182)\end{array}$ & Tamoxifen $(n=189)$ \\
\hline $9.9(8.7-11.8)$ & $5.8(5.3-8.1)$ \\
\hline 45 & 43 \\
\hline $37.2(29.2-45.5)$ & $43.3(32.8-51.6)$ \\
\hline Exemestane $(n=49)$ & Anastrozole $(n=5 I)$ \\
\hline $6.1(2.52-9.65)$ & I2.I (7.34-I6.79) \\
\hline 48.3 (18.3-78.3) & $19.9(15.32-24.46)$ \\
\hline $2.0(1.17-2.84)$ & $4.4(2.34-6.47)$ \\
\hline
\end{tabular}

\section{Adjuvant setting}

Paridaens et al

$$
\mathrm{CR}+\mathrm{PR}, \%,(95 \% \mathrm{Cl})
$$

Median TTP, weeks

Chia et $\mathrm{al}^{5}$

$$
\text { EFECT trial }
$$

$$
\begin{aligned}
& C R+P R, \% \\
& C R+P R+S D \geq 24 \text { weeks, } \%
\end{aligned}
$$

Median TTP, months

Median duration of response (from the

date of random assignment), months

Median duration of response (measured 5.5

from date of first response), months

Phase III trial by EORTC

Median PFS, months

OS, \%

Median OS, months

Llombart-Cussac et al'

\section{Bliss et al $^{17}$}

phase II randomized trial

Median TTP after first line aromatase

inhibitor therapy, months

Median OS, months

TTP after second line aromatase

inhibitor therapy, months

$$
2.0(1.17-2.84)
$$

Intergroup exemestane study

DFS first events, \%

OS, \%

Time to distant recurrence, $\%$

CLBC, \%

Van de Velde et al $^{12}$ TEAM trial

DFS at 5 years (intention to treat

$$
\text { analysis), \% }
$$

\section{OS at 5 years, \%}

RFS events at 5 years, \%

Mamounas et al $^{18} \quad$ NSABP B-33 trial

$$
4 \text { year DFS, \% }
$$

4 year RFS, \%

Neoadjuvant setting

Ellis et $\mathrm{al}^{22} \quad$ ZIO3I trial

CR, \%
PR, \%
NC, \%
DP, \%
ITT clinical response rate, (range) \%

\section{Chemoprevention}

MAP 3 trial

Number of cases

Tamoxifen followed by Tamoxifen $(n=2305)$

Exemestane $(n=2294)$

23.1 27

$15.3 \quad 17.6$

$15 \quad 17.6$

I.8 2.5

Tamoxifen followed by Exemestane $(n=4898)$

exemestane $(n=4868)$

$\begin{array}{llll}85 & 86 & \text { HR } 0.97(0.88-1.08) & \text { NS } \\ 91 & 91 & \text { HR I.00 }(0.89-1.14) & \text { NS } \\ \text { II } & 10 & \text { HR } 0.94(0.83-1.06) & \text { NS } \\ \text { Exemestane }(n=783) & \text { Placebo }(n=779) & & \\ 91 & 89 & \text { RR } 0.68 & 0.07 \\ 96 & 94 & \text { RR } 0.44 & 0.004\end{array}$

Exemestane Letrozole Anastrozole

$(n=124) \quad(n=127) \quad(n=123)$

$21.8 \quad 21.3 \quad 17.9$

$41.1 \quad 53.5 \quad 51.2$

$\begin{array}{lll}22.6 & 15.7 & 16.3\end{array}$

$\begin{array}{lll}6.5 & 4.7 & 7.3\end{array}$

$62.9(53.8-71.4) \quad 74.8(66.3-82.1) \quad 69.1(60.1-77.1)$

Exemestane $(\mathrm{n}=2285) \quad$ Placebo $(\mathrm{n}=2275) \quad$ Ratio value

$\begin{array}{llll}\text { II HR 0.35(0.18-0.7) } & 0.002\end{array}$

Abbreviations: CR, complete response; PR, partial response; SD, stable disease; TTP, time to progression; OR, odds ratio; HR, hazard ratio; PFS, progression free survival; DFS, disease free survival; RFS, recurrence free survival; OS, overall survival; CLBC, contralateral breast cancer; NC no change; DP, disease progression; ITT, intention to treat 
cancer following progression on a nonsteroidal aromatase inhibitor. ${ }^{5,6}$ Most patients were at third or subsequent lines of hormone therapy, due to tamoxifen preceding the nonsteroidal aromatase inhibitor. Therefore, as anticipated, overall response rates, defined as complete or partial response on RECIST (Response Evaluation Criteria In Solid Tumors) criteria, were low at $7.4 \%$ and $6.7 \%$ for fulvestrant and exemestane, respectively, yet clinical benefit (complete or partial response or stable disease at 24 weeks) was reported in one third of each of the treatment groups. Median time to progression was 3.7 months in both arms, with no difference in median duration of clinical benefit, supporting the use of either agent in this setting in metastatic disease.

A further trial investigating the addition of a drug to exemestane in the metastatic setting was identified by the search criteria. The BOLERO (Breast Cancer Trials of Oral Everolimus) 2 trial investigated the addition of everolimus, an inhibitor of mammalian target of rapamycin (mTOR), to exemestane in patients who had progressed on a nonsteroidal aromatase inhibitor. ${ }^{7}$ Resistance to endocrine therapy has been linked to activation of the mTOR pathway, so dual inhibition was hypothesized to offset this resistance. The trial randomized 724 postmenopausal women with advanced breast cancer, $84 \%$ of whom had hormone-sensitive disease. The primary end point of the study was progression-free survival, and the results showed that exemestane alone was inferior to the combination with everolimus, with progression-free survival of 4.1 months compared with 10.6 months (hazard ratio for the combination arm $0.36 ; 95 \%$ confidence interval [CI] $0.27-0.47, P<0.001)$. This pivotal study showed a definite objective clinical benefit, supporting the biological concept of crosstalk between the two signaling pathways.

\section{First-line use}

A large-scale multicenter, multinational Phase III trial was conducted by the EORTC (European Organisation for Research and Treatment of Cancer) to assess the efficacy of using exemestane or tamoxifen as first-line therapy for metastatic breast cancer $(n=371)$, following on from the low incidence of toxicity in the Phase II trial. ${ }^{8-10}$ Both drugs were found to be well tolerated and participants treated with exemestane had a significantly higher response rate than those treated with tamoxifen ( $46 \%$ versus $31 \%, P=0.005$ ). Both treatments had equivalent median overall survival. There was also a significantly longer median progression-free survival with exemestane (9.9 months, 95\% CI 8.7-11.8) compared with tamoxifen (5.8 months, 95\% CI 5.3-8.1, $P=0.028$ ) but this did not translate into a statistically significant long-term benefit in progression-free survival at a later follow-up of 46 months. While the nonsteroidal aromatase inhibitors (anastrozole and letrozole) are now recognized as the firstline endocrine therapy of choice in the setting of metastatic breast cancer, the results of this study provide evidence that a steroidal aromatase inhibitor also works in the same setting and is more efficacious than tamoxifen.

A smaller scale Phase II trial, published in 2012, assessed the efficacy of using upfront exemestane or anastrozole (a nonsteroidal aromatase inhibitor) in first-line treatment for metastatic ER-positive breast cancer, with participants permitted to switch to the other aromatase inhibitor at progression. ${ }^{11}$ Half of the 103 participants had previously received tamoxifen and $60 \%$ had received chemotherapy. No significant difference in clinical outcome between the two treatments was found and both drugs were well tolerated overall, with no serious adverse events reported. The authors concluded that there was insufficient evidence to support one agent as being more effective than the other or to justify a subsequent Phase III trial.

\section{Summary}

In metastatic breast cancer, exemestane has been demonstrated to be superior to megesterol acetate, following progression on tamoxifen, and also to tamoxifen, as a first-line therapy. There is also evidence to support noninferiority to nonsteroidal aromatase inhibitors and the pure antiestrogen, fulvestrant.

\section{Adjuvant setting}

Exemestane is licensed in the adjuvant setting as a sequential treatment after 2-3 years of tamoxifen in postmenopausal patients with ER-positive tumors. This section divides adjuvant treatment into three categories, based on the timing of randomization to exemestane or an alternative drug in three key Phase III trials, as follows.

\section{Upfront therapy}

The Phase III TEAM (Tamoxifen Exemestane Adjuvant Multinational) trial randomized patients to upfront exemestane for 5 years versus a switch to exemestane after 2.5-3 years of tamoxifen. ${ }^{12}$ Postmenopausal women (median age 64 [range 35-96] years) with ER-positive breast cancer were randomly assigned in a 1:1 ratio. At 5 years, no differences were noted in disease-free survival and overall survival between the groups, indicating that steroidal aromatase inhibitors alone are an option in adjuvant treatment. This is the only study in the adjuvant setting to assess exemestane upfront.

A prospective biomarker substudy of the same TEAM trial tested the hypothesis that patients with low progesterone 
Table 2 Summary of trials assessing the safety of exemestane

\section{Safety \\ Metastatic trials}

Paridaens et al ${ }^{10}$

\author{
Phase III trial by EORTC
}

\section{Nonhematologic events}

Alopecia

Anorexia

Anxiety

Arthralgia

Cardiac dysrhythmia

Cardiac dysfunction

Constipation

Cough

Deep vein thrombosis

Depression

Diarrhea

Dyspnea

Edema

Fatigue/malaise/lethargy

Gastrointestinal events

Genitourinary events

Hot flashes

Hypertension

Infection

Insomnia

Musculoskeletal events

Nausea

Neurologic, dizziness

Neurologic, sensory

Pain, bone

Pain, other

Phlebitis

Skin

Sweating

Vaginal bleeding

Vaginal discharge

Vomiting

Weight loss

Weight gain

Hematologic events

Leukopenia

Neutropenia

Thrombocytopenia

Anemia

Biochemical changes

Creatinine

Bilirubin

AST

ALT

Kaufmann et al'

Any adverse event

Hot flashes

Nausea

Fatigue

Increased sweating

Insomnia

$\begin{array}{llll}\begin{array}{l}\text { Exemestane } \\ (n=182)\end{array} & \begin{array}{l}\text { Tamoxifen } \\ (n=189)\end{array} & \\ \text { Grade I/2 (\%) } & \text { Grade 3/4 (\%) } & \text { Grade I/2 (\%) } & \text { Grade 3/4 (\%) }\end{array}$

3.8

10.4

II

II.5

5.4

4.4

8.2

14.8

1.6
7.7

7.7

8.8

13.7

9.9

35.7

8.2

4.9

34.6

8.2

15.4

9.9

4.9

17

11.5

8.8

29.1

31.9

0

I I

10.4

I.I

2.2

8.2

16.5

17.6

17.6

9.9

4.4

28

7.1

11.5

49.5

56.6

Exemestane

( $n=358), \%$

39.1

12.6

9.2

7.5

4.5

3.6

\section{0}

0.5

0

0

1.6

0.5

0

0

0

0.5

0

1.6

I.I

I.I

0

0

0.5

3.3

I.I

0

0.5
0

0

0.5

3.8

2.7

0

0.5

0

0

0

0

0.5

I.I

\section{0}

1.1

1.6

I.I

1.6

3.3

4.9

7.7

Megestrol acetate

( $n=400), \%$

45.8

5

5

10.3

7.5

3.3
I.I 0

$9.5 \quad 1.1$

$7.4 \quad 0$

$5.3 \quad 0$

I.6 I.I

$3.2 \quad 0.5$

$12.2 \quad 0$

$15.9 \quad 0$

I.6 0.5

$\begin{array}{ll}6.9 & 0.5\end{array}$

$2.6 \quad 0$

$13.2 \quad 2.6$

$10.1 \quad 1.1$

$35.4 \quad$ I.I

$11.6 \quad 0.5$

$5.3 \quad 0$

$38.1 \quad 0$

$2.6 \quad 3.2$

12.20

$5.3 \quad 0$

$0.5 \quad$ I.I

$19 \quad 0.5$

10.10

$9.5 \quad 1.1$

$29.1 \quad 5.8$

$\begin{array}{ll}0.5 & 0.5\end{array}$

$9.5 \quad 0$

90

$3.2 \quad 0.5$

6.90

$6.9 \quad 0$

$14.8 \quad$ I.I

$12.2 \quad 0.5$

$18 \quad 0$

$13.8 \quad 0$

$11.6 \quad 0.5$

$34.9 \quad 1.1$

$4.2 \quad 0$

$\begin{array}{ll}1.1 & 1.6\end{array}$

$\begin{array}{ll}50.8 & 4.8\end{array}$

$46 \quad 4.2$

OR $95 \% \mathrm{Cl}$

(EXE/MA)

$0.76 \quad 0.576-1.02$

$2.73 \quad 1.58-4.72$

$1.93 \quad 1.09-3.43$

$0.71 \quad 0.43-1.19$

$0.58 \quad 0.3 \mathrm{I}-1.08$

I.12 $0.5 \mathrm{I}-2.45$
$27.5 \quad 3.2$

(Continued) 
Table 2 (Continued)

\begin{tabular}{|c|c|c|c|c|c|c|}
\hline & Dizziness & 3.4 & 3 & 1.12 & $0.5-2.53$ & \\
\hline & Increased appetite & 2.8 & 5.8 & 0.47 & $0.22-1$ & \\
\hline & Abdominal pain & 2.8 & 4.3 & 0.65 & $0.29-1.43$ & \\
\hline & Vomiting & 2.8 & 0.8 & 3.8 & $1.04-13.93$ & \\
\hline & Rash & 2 & 0 & & & \\
\hline & Constipation & 0.8 & 2.5 & 0.33 & $0.09-1.21$ & \\
\hline & Dyspnea & 0.3 & 3 & 0.09 & $0.01-0.7$ & \\
\hline & $\begin{array}{l}\text { Withdrawal from study } \\
\text { due to adverse events }\end{array}$ & 1.7 & 5 & $P=0.011$ & & \\
\hline \multicolumn{7}{|l|}{ Adjuvant setting } \\
\hline \multirow[t]{41}{*}{ Bliss et al ${ }^{17}$} & & $\begin{array}{l}\text { Tamoxifen followed } \\
\text { by exemestane } \\
(\mathrm{n}=2105), \%\end{array}$ & $\begin{array}{l}\text { Tamoxifen } \\
(n=2036), \%\end{array}$ & OR & $99 \% \mathrm{Cl}$ & $P$ value \\
\hline & $\begin{array}{l}\text { All cardiovascular events } \\
\text { (excluding hypertension and VTE) }\end{array}$ & 12.3 & 10.4 & 1.21 & $0.94-1.57$ & 0.049 \\
\hline & Ischemic cardiovascular disease & 6 & 4.6 & 1.33 & $0.92-1.92$ & 0.043 \\
\hline & Angina & 5.2 & 3.9 & 1.37 & $0.92-2.05$ & 0.038 \\
\hline & Other cardiovascular event & 6.1 & 5.4 & 1.15 & $0.8 \mathrm{I}-\mathrm{I} .65$ & 0.284 \\
\hline & Hypertension & 26.7 & 23.3 & 1.2 & $0.99-1.45$ & 0.011 \\
\hline & VTE & 1 & 0.9 & 1.02 & $0.42-2.49$ & 0.955 \\
\hline & DVT & 0.8 & 0.9 & 0.91 & $0.35-2.33$ & 0.788 \\
\hline & Fractures & 6.8 & 5.7 & 1.2 & $0.86-1.69$ & 0.147 \\
\hline & Arthritis (all types) & 8.7 & 8.2 & 1.07 & $0.80-1.44$ & 0.534 \\
\hline & Osteoarthritis & 5.7 & 5.8 & 0.97 & $0.68-1.39$ & 0.844 \\
\hline & Carpal tunnel syndrome & 0.5 & 0.3 & 1.38 & $0.35-6.11$ & 0.509 \\
\hline & Osteoporosis & 5 & 4.7 & 1.07 & $0.73-1.57$ & 0.632 \\
\hline & Muscle cramps & 0.6 & 0.7 & 0.84 & $0.28-2.40$ & 0.64 \\
\hline & Pain, musculoskeletal & 15 & 12.8 & 1.2 & $0.95-1.52$ & $0.04 I$ \\
\hline & Pain, limb and/or foot & 2.7 & 2.4 & 1.13 & $0.67-1.92$ & 0.54 \\
\hline & Arthralgia & 6.4 & 6.4 & 1 & $0.72-|.4|$ & 0.97 \\
\hline & Myalgia & 0.9 & 0.5 & 1.59 & $0.56-4.87$ & 0.225 \\
\hline & Pain, abdominal & 2.3 & 1.5 & $1.5 \mathrm{I}$ & $0.8 I-2.86$ & 0.075 \\
\hline & Pain, other & 5.5 & 6.1 & 0.9 & $0.63-1.28$ & 0.425 \\
\hline & Serious gynecologic events & 1.5 & 1.8 & 0.83 & $0.42-1.62$ & 0.451 \\
\hline & Vaginal bleeding & 0.8 & 1.2 & 0.62 & $0.25-1.46$ & 0.129 \\
\hline & $\begin{array}{l}\text { Uterine polyps/fibroids and } \\
\text { endometrial hyperplasia }\end{array}$ & 0.9 & 1.7 & 0.56 & $0.23-1.26$ & 0.052 \\
\hline & Uterine polyps/fibroids & 0.8 & 1.6 & 0.53 & $0.2 \mathrm{I}-\mathrm{I} .24$ & 0.043 \\
\hline & Endometrial hyperplasia & 0.1 & 0.2 & 0.49 & $0.02-5.91$ & 0.408 \\
\hline & Vaginal discharge & 0.4 & 0.6 & 0.59 & $0.15-2.03$ & 0.242 \\
\hline & Menopausal events & 11.6 & $1 \mathrm{I} .4$ & 1.02 & $0.79-1.32$ & 0.844 \\
\hline & Hot flashes & 9 & 8.8 & 1.02 & $0.76-1.36$ & 0.876 \\
\hline & Anxiety & 1.7 & 1.3 & 1.29 & $0.65-2.63$ & 0.313 \\
\hline & Depression & 4.9 & 4.6 & 1.07 & $0.73-1.59$ & 0.622 \\
\hline & Diarrhea & 0.8 & 0.8 & 0.97 & $0.37-2.52$ & 0.922 \\
\hline & Dizziness & 3.2 & 3.9 & 0.83 & $0.53-1.29$ & 0.259 \\
\hline & Fatigue & 6.6 & 6.7 & 0.97 & $0.70-1.35$ & 0.823 \\
\hline & Gl ulcer & 0.9 & 0.3 & 2.92 & $0.86-13.1$ & 0.018 \\
\hline & Headaches & 3.9 & 4.7 & 0.84 & $0.56-1.26$ & 0.252 \\
\hline & Hypercholesterolemia & 5.1 & 4.9 & 1.04 & $0.7|-| .5 \mid$ & 0.8 \\
\hline & Insomnia & 6.3 & 6.2 & 1.01 & $0.72-1.42$ & 0.913 \\
\hline & Nausea & 1.7 & 1.5 & 1.16 & $0.6-2.3$ & 0.543 \\
\hline & Paresthesia & 0.4 & 0.3 & 1.45 & $0.33-7.33$ & 0.477 \\
\hline & Polypectomy & 0.1 & 0.2 & 0.39 & $0.02-3.8$ & 0.238 \\
\hline & Sweating & 3.7 & 3.8 & 0.95 & $0.62-1.47$ & 0.769 \\
\hline Van de Velde et al ${ }^{12}$ & & $\begin{array}{l}\text { Tamoxifen followed } \\
\text { by exemestane } \\
(n=48 \mid 4), \%\end{array}$ & $\begin{array}{l}\text { Exemestane } \\
(\mathrm{n}=4852), \%\end{array}$ & $P$ value & & \\
\hline
\end{tabular}


Table 2 (Continued)

\begin{tabular}{|c|c|c|c|}
\hline \multicolumn{4}{|l|}{ Cardiac disorders } \\
\hline Arrhythmia & 3 & 4 & 0.038 \\
\hline Cardiac failure & $<1$ & 1 & 0.009 \\
\hline Myocardial ischemia or infarction & 1 & 2 & 0.171 \\
\hline Other cardiac disorders & 2 & 2 & 0.843 \\
\hline \multicolumn{4}{|l|}{ Vascular disorders } \\
\hline Embolism & I & $<1$ & 0.808 \\
\hline Flushes and sweats & 40 & 35 & $<0.0001$ \\
\hline Hypertension & 5 & 6 & 0.0003 \\
\hline Peripheral arterial disease & $<1$ & $<1$ & 0.038 \\
\hline Venous thrombosis & 2 & $<1$ & $<0.0001$ \\
\hline Other vascular events & 4 & 3 & 0.024 \\
\hline \multicolumn{4}{|l|}{ Reproductive system } \\
\hline \multicolumn{4}{|l|}{ and breast disorders } \\
\hline Breast or nipple disorder & 5 & 6 & 0.27 \\
\hline Endometrial abnormalities & 4 & $<1$ & $<0.0001$ \\
\hline Genital or vaginal discharge & 8 & 3 & $<0.0001$ \\
\hline Postmenopausal bleeding & 5 & 3 & $<0.0001$ \\
\hline Vaginal dryness & 6 & 7 & 0.038 \\
\hline Vulvovaginal disorders & 5 & 2 & $<0.0001$ \\
\hline $\begin{array}{l}\text { Other reproductive system } \\
\text { and breast disorders }\end{array}$ & 3 & 2 & $<0.000$ I \\
\hline \multicolumn{4}{|l|}{ Musculoskeletal and } \\
\hline Fractures & 3 & 5 & $<0.0001$ \\
\hline Joint disorders & 31 & 36 & $<0.0001$ \\
\hline Muscle disorders & 13 & 11 & 0.0014 \\
\hline Osteoporosis & 6 & 10 & $<0.0001$ \\
\hline $\begin{array}{l}\text { Other musculoskeletal and } \\
\text { connective tissue disorders }\end{array}$ & 13 & 15 & 0.0023 \\
\hline \multicolumn{4}{|l|}{ Nervous system disorders } \\
\hline Cerebrovascular insufficiency, & I & 2 & 0.035 \\
\hline \multicolumn{4}{|l|}{ infarction, or thrombosis } \\
\hline Dizziness & 6 & 5 & 0.051 \\
\hline Headache & 8 & 8 & 0.97 \\
\hline Nerve compression disorders & 2 & 3 & 0.008 \\
\hline Other nervous system disorders & 14 & 17 & 0.0004 \\
\hline \multicolumn{4}{|l|}{ Psychiatric disorders } \\
\hline Depression & 9 & 9 & 0.583 \\
\hline Reduction or loss of libido & 3 & 4 & 0.061 \\
\hline Sleep disorder or insomnia & 10 & 13 & $<0.0001$ \\
\hline Other psychiatric disorders & 9 & 8 & 0.736 \\
\hline \multirow{2}{*}{\multicolumn{4}{|c|}{$\begin{array}{l}\text { Laboratory investigations, and } \\
\text { metabolism and nutrition disorders }\end{array}$}} \\
\hline & & & \\
\hline Abnormal liver function tests & 3 & 4 & 0.038 \\
\hline Hyperlipidemia & 3 & 5 & $<0.0001$ \\
\hline Weight increased & 9 & 7 & 0.005 \\
\hline Other investigations & 6 & 7 & 0.068 \\
\hline Other metabolism and & 9 & & 0.051 \\
\hline \multicolumn{4}{|l|}{ nutrition disorders } \\
\hline \multicolumn{4}{|l|}{ Other disorders } \\
\hline Endocrine disorders & 2 & 3 & 0.028 \\
\hline \multirow[t]{3}{*}{ Renal and urinary disorders } & 5 & 4 & 0.006 \\
\hline & Exemestane & Placebo & $P$ value \\
\hline & $(n=2240), \%$ & $(n=2248), \%$ & \\
\hline Any & 88 & 85 & 0.003 \\
\hline Cardiac: hypertension & 15 & 16 & 0.65 \\
\hline
\end{tabular}

Chemoprevention

Goss et al ${ }^{26}$

Cardiac: hypertension

\section{Cardiac disorders}

Other cardiac disorders 2

Vascular disorders

40

Hypertension

Peripheral arterial disease

bosis

Reproductive system

and breast disorders

Breast or nipple disorder

Postmenopausal bleeding

Vaginal dryness

Vulvovaginal disorders

and breast disorders

Musculoskeletal and

connective tissue disorders

Fractures

Joint disord

Other musculoskeletal and

Cerebrovascular insufficiency,

infarction, or thrombosis

Dizziness

Nerve co

Other nervous system disorders

Reduction or loss of libido

Sleep disorder or insomnia

Abnormal liver function tests

Hyperlipidemia

Other metabolism and

nutrition disorders

Other disorders

Endocrine disorders

Renal and urinary disorders

5

(Continued) 
Table 2 (Continued)

\begin{tabular}{llll}
\hline Endocrine & & 32 & $<0.001$ \\
Hot flashes & 40 & 21 & 0.03 \\
Fatigue & 23 & 19 & 0.046 \\
Sweating & 22 & 8 & 0.04 \\
Insomnia & 10 & & 0.002 \\
Constitutional and gastrointestinal & 5 & 3 & 0.06 \\
Diarrhea & 15 & 13 & 0.04 \\
Heartburn & 7 & 5 & 0.01 \\
Nausea & 11 & 9 & 0.32 \\
Musculoskeletal: arthritis & & 9 & 0.96 \\
Neurologic & 8 & 10 & 0.45 \\
Dizziness & 11 & & 0.054 \\
Mood alteration or depression & & 10 & 0.04 \\
Pain & 9 & 5 & 0.01 \\
Back & 7 & 27 & 0.14 \\
Extremity & 30 & 9 & 0.68 \\
Joint & 7 & 12 & 0.72 \\
Muscle & 10 & 15 & 0.39 \\
Upper respiratory: cough & 16 & & 0.78 \\
Sexual function: vaginal dryness & 16 & 6.4 & 0.58 \\
Secondary end point toxic effects & 6.7 & 1.3 & \\
Clinical skeletal fracture & 1.7 & 4.9 & 1.7 \\
New osteoporosis & 4.7 & & \\
Cardiovascular events & 1.9 & & \\
Other solid tumors or & & & \\
hematologic malignant lesions & & & \\
\hline
\end{tabular}

Abbreviations: ALT, alanine transaminase; AST, aspartate transaminase; DVT, deep vein thrombosis; EORTC, European Organisation for Research and Treatment of Cancer; OR, odds ratio; $\mathrm{Cl}$, confidence interval; $\mathrm{Gl}$, gastrointestinal; MA, megestrol acetate; $\mathrm{EXE}$, exemestane; VTE, venous thromboembolism.

receptor expression have increased clinical benefit from an aromatase inhibitor compared with tamoxifen, this being an association identified in the ATAC (Arimidex Tamoxifen Alone or in Combination) adjuvant trial. ${ }^{13} \mathrm{~A}$ treatment benefit with exemestane was not identified in the progesterone receptor-poor patients. However, on multivariate analysis, the relative risk of relapse increased proportionally with decreasing expression of either receptor and there was a trend for increased treatment benefit in patients with ERrich tumours. ${ }^{14}$

\section{Sequential therapy}

IES (the Intergroup Exemestane Study) enrolled 4742 postmenopausal patients with ER-positive or unknown receptor breast cancer, who had received 2-3 years of treatment with tamoxifen. ${ }^{15,16}$ Participants were randomized to either continue tamoxifen treatment or to switch to exemestane to complete a 5-year treatment course, the median follow-up period being 30.6 months. The randomization method differed from that of TEAM in that this did not occur upfront, but after 2-3 years of endocrine therapy. An absolute reduction in risk of death of $4.7 \%$ was found in the switch arm, with an adjusted hazard ratio of 0.68 (95\% CI 0.56-0.82; $P<0.001)$ comparing against the tamoxifen arm. There was no significant difference in overall survival between the two treatment arms, with 93 and 106 deaths in the switch and tamoxifen arms, respectively. However, participants in the switch arm had a significantly lower incidence of the secondary end point, ie, contralateral breast cancer ( 9 versus 20 cases, $P=0.04)$.

A recent update at median follow-up of 7.6 years showed the persistent efficacy of the sequential arm in disease-free survival, comparing against tamoxifen. ${ }^{17}$ The switch arm also demonstrated a modest but significant survival benefit (352 deaths) compared with the tamoxifen (405 deaths) arm (hazard ratio $0.86 ; 95 \%$ CI $0.75-0.99 ; P=0.04$ ).

\section{Extended adjuvant therapy}

The NSABP (National Surgical Adjuvant Breast and Bowel Project)-B33 trial investigated the potential value of extended adjuvant endocrine therapy using exemestane. ${ }^{18}$ Until recently, when the long-term results of the ATLAS (Adjuvant Tamoxifen Longer Against Shorter) trial were published (showing that 10 years of tamoxifen produced superior benefits in terms of disease-free survival when compared with 5 years), it was thought that extending adjuvant tamoxifen beyond 5 years would not produce any significant impact, given that a second peak of recurrence occurs after 
5 years. ${ }^{19,20}$ In consequence, researchers started to explore the potential use of an aromatase inhibitor following 5 years of adjuvant tamoxifen. A similar National Cancer Institute of Canada study, Mammary 17 (MA17), looking into a different aromatase inhibitor, ie, letrozole, was also started around the same time. ${ }^{21}$

In the NASBP-B33 trial, postmenopausal patients with clinically staged T1-3 N1M0 breast cancer who were disease-free after 5 years of tamoxifen were randomly assigned to 5 years of exemestane or 5 years of placebo. However, in October 2003, the B-33 trial terminated accrual following publication of the results from MA17, which demonstrated the benefit of letrozole, a third-generation nonsteroidal aromatase inhibitor, in a similar role as for extended endocrine therapy after tamoxifen. At the time when B-33 trial terminated, a total of 1598 participants of the planned 3000 had been recruited. Despite premature closure and crossover to exemestane by a substantial proportion of patients, following unblinding of the participants, the intention-to-treat analysis of the original exemestane assignment demonstrated a trend for improvement in disease-free survival and a significant improvement in relapse-free survival of $96 \%$ versus $94 \%$ at a median follow-up of 30 months, the latter defined as time to recurrence or contralateral breast cancer.

\section{Summary}

Overall, a switch to exemestane appears to improve diseasefree survival compared with tamoxifen alone. Five years of an aromatase inhibitor has not been demonstrated to be superior to sequential treatment at this time, but it appears to be noninferior to 5 years of tamoxifen and an acceptable alternative in patients for whom tamoxifen is contraindicated. There may also be a role for extended adjuvant treatment using this class of drug following on from 5 years of tamoxifen.

\section{Neoadjuvant setting}

The Phase II ACOSOG (American College of Surgeons Oncology Group) Z1031 trial investigated the efficacy of exemestane, anastrozole, and letrozole as neoadjuvant therapy in 377 postmenopausal patients with ER-positive, stage II-III breast cancer. ${ }^{22}$ Participants were randomized to receive one of the three aromatase inhibitors for 16-18 weeks before surgery. The results show that the overall breast conservation rate was $68 \%$, which is comparable with that in the NSABP B18 study addressing the efficacy of neoadjuvant chemotherapy, including doxorubicin and cyclophosphamide. ${ }^{23}$
Of the 124 participants randomized to the exemestane arm in the Z1031 trial, 27 had a complete response, 51 had a partial response, 28 had stable disease, and eight had progressive disease. Five participants discontinued the medication and measurements were unavailable for five patients. The highest clinical response rate was found in the letrozole arm (rate 70.9\%; 95\% CI 62.2-78.6) followed by the anastrozole arm (rate 66.7\%; 95\% CI 57.6-74.9) and exemestane arm (rate $60.5 \%$; 95\% CI 51.3-69.1), but there was no significant difference between the three arms. Based on this trial, all three aromatase inhibitors could be considered for further investigation in a Phase III trial for a head-to-head comparison with chemotherapy as the neoadjuvant therapy.

Other smaller studies have also shown an objective reduction in tumor size with aromatase inhibitors, and celecoxib has again been trialed in combination with exemestane. ${ }^{24}$ Aromatase inhibitors are likely to have an increasing role in the future in selected patients with locally advanced breast cancer.

\section{Summary}

The use of exemestane as neoadjuvant therapy is an attractive alternative to chemotherapy. Data exist showing increased breast conservation rates, but long-term outcome data, as well as a head-to-head comparison against chemotherapy, are not yet available. Whether exemestane is a superior or an inferior aromatase inhibitor in this setting remains to be established.

\section{Chemoprevention}

Most of the evidence for the efficacy of treatment in the reduction of breast cancer risk comes from the selective estrogen receptor modulators, ie, tamoxifen and raloxifene. A meta-analysis suggested an overall reduction in the risk of invasive breast cancer of 38\% comparing tamoxifen against placebo, with no difference in overall survival. The number needed to treat to prevent one breast cancer at 5 years is 95 with tamoxifen. ${ }^{25}$ However, long-term adherence to tamoxifen is poor in those individuals who are "at risk" because of concerns about the uncommon but serious adverse effects of venous thromboembolism and endometrial malignancy. The superiority of aromatase inhibitors over tamoxifen in terms of efficacy, notably in reducing contralateral cancers in the adjuvant trials as mentioned, has helped to drive exploration of their role in chemoprevention.

The large-scale, double-blind, placebo-controlled Mammary Prevention Trial 3 (MAP 3) was set up by the National Cancer Institute of Canada to assess exemestane as a chemoprevention agent. ${ }^{26} \mathrm{~A}$ total of 4560 postmenopausal patients with at least one risk factor for future breast 
cancer took part and, at a median follow-up of 3 years, there were significantly fewer breast cancer occurrences in the exemestane arm, with 11 versus 32 . The number needed to treat to prevent one case of breast cancer was 94, but this was projected to decrease to 26 at 5 years.

\section{Summary}

Exemestane appears to be efficacious in the setting of chemoprevention when compared with placebo. Long-term follow-up is awaited, and its specific role when compared with other aromatase inhibitors and selective estrogen receptor modulators remains to be elucidated.

\section{Safety}

Many of the trials investigating the efficacy of exemestane in the adjuvant and metastatic settings as well as in chemoprevention included safety and quality of life as a secondary end point, and provide valuable information. The adjuvant trials have the advantage of absence of metastatic disease, which can confound the causality between treatments and outcomes. Furthermore, both adjuvant and chemoprevention trials, as opposed to metastatic studies, have an additional advantage in long-term follow-up, which is more suitable to assess safety of treatments (Table 2).

\section{Metastatic trials}

In the metastatic setting, the EORTC trial of exemestane versus tamoxifen as first-line treatment provides valuable data. $^{8-10}$ Both drugs were well tolerated and there were no treatment-related deaths. Sixty-one patients had a grade 3/4 nonhematological adverse events in the tamoxifen arm versus 41 with exemestane. With exemestane, there were reduced grade $2 / 3$ hot flashes $(6.5 \%$ versus $12.2 \%)$ and grade $1 / 2$ vaginal discharge and bleeding (3.3\% versus $10.1 \%)$, but increased grade $1 / 2$ arthralgias ( $11.5 \%$ versus $5.3 \%$ ), grade $1 / 2$ cardiac dysrhythmias $(5.4 \%$ versus $1.6 \%)$, and grade 1 diarrhea (8.8\% versus $2.6 \%$ ). Approximately one-third of patients in each arm had significant fatigue and one-fifth had nausea. There was no detrimental effect on cholesterol levels or on atherogenic indices, which are well known risk factors for coronary artery disease, ${ }^{27}$ in patients with exemestane.

The second trial to be discussed assessed exemestane versus megestrol acetate in metastatic breast cancer. ${ }^{1}$ This trial provides safety data in relation to a comparison with megestrol acetate, which cannot be obtained in adjuvant and chemoprevention settings when the drug is not being used. Already demonstrated to be clinically more effective in the quality of life assessment, patients treated with exemestane showed a statistically significant improvement in physical functioning, role functioning, global health, fatigue, dyspnea, and constipation compared with patients who received megestrol acetate.

\section{Adjuvant trials}

In IES, acute effects of arthralgias and diarrhea were more common with exemestane than with tamoxifen, with fewer gynecological symptoms, muscle cramps, and thromboembolic events. ${ }^{28,29}$

The incidence of adverse events decreased over a longer follow-up period due to the cessation of treatment. ${ }^{29}$ For exemestane, the incidence of musculoskeletal pain, arthritis, and osteoporosis decreased. However, there was an objective decrease in bone mineral density compared with baseline in the patients who switched to exemestane, which was more marked in the first 6 months than at 2 years. No patients developed osteoporosis, but there was a significantly increased fracture risk of $7 \%$ versus $5 \%$ (odds ratio $1.45,95 \%$ CI 1.13-1.87, $P=0.003) .{ }^{30}$ Importantly, the increased fracture incidence in the exemestane arm did not persist at follow-up, and mean bone mineral density returned to pretreatment values at one year after discontinuation. Smaller trials have addressed the incidence of reduced bone mineral density compared with tamoxifen or placebo and, similarly, an early objective loss is seen, but is not maintained in the follow-up period. ${ }^{31,32}$

Similarly, the increased risk of serious gynecological events with tamoxifen did not continue into the follow-up period, with some objective reversal of endometrial thickening. ${ }^{33}$ At a median follow-up of 91 months, there was an increased incidence of carpal tunnel syndrome with exemestane at $2.8 \%$ versus $0.6 \%$ with tamoxifen (OR 5.23, $99 \%$ CI 2.39-11.49, $P<0.0001$ ), warranting surgical intervention in $69 \%$ of cases. ${ }^{34}$

There was a trend towards increased cardiovascular events (excluding hypertension and venous thromboembolism) following sequential treatment (tamoxifen followed by exemestane) as opposed to 5 years of tamoxifen alone, at $12.3 \%(n=259)$ versus $10.4 \%(n=211)$, which did not reach statistical significance but which the authors felt was important to acknowledge. However, despite the reported increase in cardiovascular morbidity, this did not equate to a higher cardiovascular death rate.

Follow-up for IES found what is desirable for any adjuvant treatment, ie, a prolongation of risk reduction, without prolongation of adverse effects.

The TEAM trial, which is the largest adjuvant study of exemestane to date, found that sequential treatment (tamoxifen 
for 2-3 years before exemestane) had a higher incidence of gynecological symptoms ( $20 \%$ versus $11 \%$ ), venous thrombosis ( $2 \%$ versus $1 \%)$, and endometrial abnormalities ( $4 \%$ versus $<1 \%$ ) compared with 5 years of exemestane alone. Musculoskeletal adverse events, including arthralgias and myalgias ( $50 \%$ versus $44 \%$ ), hypertension ( $6 \%$ versus $5 \%$ ), and hyperlipidemia ( $5 \%$ versus $3 \%$ ) were reported more frequently with exemestane alone. ${ }^{12,35,36}$ Rather than exemestane causing an increase in lipids per se, substudies of TEAM have found exemestane to have a neutral effect on lipid profiles, compared with the beneficial effect found with tamoxifen. ${ }^{37-39}$ Hot flashes were reported to be significantly increased with tamoxifen, with an increase of 33\% from baseline compared with $7 \%$ in the exemestane arm. With exemestane treatment, musculoskeletal adverse events including arthralgias and myalgias ( $50 \%$ versus $44 \%$ ), hypertension ( $6 \%$ versus $5 \%$ ), and hyperlipidemia (5\% versus $3 \%$ ) were reported more frequently. As with the IES, there was increased bone loss in the aromatase inhibitor arm. A substudy of 161 patients found that the greatest loss in bone mineral density was within the first 6 months, but this loss stabilized after 6-12 months of treatment. Tamoxifen caused a $0.5 \%$ increase in bone mineral density at the spine at 12 months, whilst exemestane-treated patients had a $2.6 \%$ decrease at 6 months with a further $0.2 \%$ decrease at 12 months $(P=0.0008)$. Bone loss was found to stabilize after 6-12 months of treatment. ${ }^{40,41}$

A substudy of the TEAM trial in Dutch participants to assess possible changes in cognitive function with each endocrine therapy produced interesting results. ${ }^{42,43}$ After one year of tamoxifen $(n=80)$ there was a statistically significant decrease in verbal recall and executive functioning. This was not found in the 99 exemestane patients assessed.

\section{Chemoprevention}

Exemestane is associated with increased serum bone turnover markers in healthy postmenopausal women, suggesting a specific bone formation effect related to its androgenic structure. ${ }^{44,45}$ A nested substudy of the MAP 3 trial investigated bone health. After 2 years of treatment with exemestane, there was more age-related bone loss in postmenopausal women than in those on placebo, despite calcium and vitamin D supplementation. To date, there has been no significant difference in skeletal-related events demonstrated, but longer-term follow-up is awaited. ${ }^{46}$ Adverse events occurred in $88 \%$ of the exemestane group versus $85 \%$ of the placebo group $(P=0.003)$ with no significant difference in cardiovascular events. There was an increased incidence of menopausal symptoms and sexual dysfunc- tion in the exemestane group, but this did not affect overall health-related quality of life.

\section{Summary}

Both tamoxifen and aromatase inhibitors (including exemestane) have differing side effect profiles, by virtue of their mechanisms of action. Tamoxifen has partial estrogen agonist properties, which have a protective effect on bone mineral density and the cardiovascular system. This cannot be said for aromatase inhibitors, and it is this depletive action on estrogen levels that is thought to have an adverse effect on cardiac and bone health.

All patients receiving aromatase inhibitors are generally recommended to have a baseline bone densitometry scan which should be repeated as appropriate. ${ }^{47}$ Intervention with bisphosphonates and bone-strengthening medication is only recommended if the patient has osteoporosis at baseline. Cardiac dysfunction is a less frequent adverse event, hypothesized to be secondary to the cardioprotective effect of tamoxifen in the control arm, rather than an increased risk caused by aromatase inhibitors per se.

More commonly seen adverse effects of aromatase inhibitors are arthralgias and myalgias that can lead to premature discontinuation of the drug. These agents are also associated with fewer hot flashes, weight gain, thromboembolic disease, and endometrial cancer than tamoxifen.

To date, the side effect profile of exemestane appears to be a class effect (as a member of the third-generation aromatase inhibitors) with no specific adverse effects demonstrated as a result of it being a steroidal aromatase inhibitor.

\section{Adherence}

Adherence to a prescribed course of medication can be influenced by many factors, including patient age, financial situation if self-funding, and the side effect profile. Most publications have addressed aromatase inhibitors as a whole, and there is a paucity of data related to adherence specifically with regard to exemestane. A retrospective review of 13,593 patients with postmenopausal breast cancer prescribed exemestane, with anastrozole or letrozole as adjuvant therapy, found nonadherence in $23 \%$ patients over one year, based upon medication possession ratio. ${ }^{48}$ Using multivariate logistic regression, factors associated with nonadherence included younger age, breast-conserving surgery rather than mastectomy, and an increased Charlson Comorbidity Index. The study recommended identifying such individuals for early supervision and to support "medication-taking behaviors". 
One prospective study recruited 503 patients with early breast cancer to receive letrozole or exemestane, and participants could switch to the other aromatase inhibitor following a 2-8-week washout period if side effects became intolerable (Table 3). ${ }^{49}$ Of all the patients, $32.4 \%$ stopped their aromatase inhibitor within 2 years because of adverse effects, $24.3 \%$ of which were musculoskeletal in nature. The median time to discontinuation for adverse effects was 6 (range 0.1-21) months, and this duration was significantly shorter in the exemestane arm than in the letrozole arm (hazard ratio 1.5; 95\% CI 1.1-2.2, $P=0.02$ ). Younger age and prior taxane exposure increased the likelihood of discontinuation of therapy. Following a switch to the other aromatase inhibitor, more than a third of patients could continue their adjuvant treatment. This may be attributable to the myalgias and peripheral paraesthesias experienced by many patients when on taxane therapy, affecting the threshold of tolerance for further adverse effects with aromatase inhibitors.

A substudy of the TEAM trial looked specifically at agespecific nonpersistence with endocrine therapy, this being defined as discontinuation of therapy within one year of follow-up. ${ }^{50}$ Conversely, increased age was associated with a higher proportion of nonpersistence at one year. This could be secondary to older patients having an increased likelihood of coexisting health problems requiring medication. This polypharmacy could affect compliance and therefore adherence to the proposed 5-year treatment.

\section{Summary}

There is clearly a lack of evidence in the literature concerning adherence to exemestane. The limited evidence available

Table 3 Adherence of early postmenopausal breast cancer patients to exemestane versus letrozole

\begin{tabular}{|c|c|c|c|c|}
\hline \multicolumn{5}{|l|}{ Adherence } \\
\hline \multirow[t]{5}{*}{ Henry et $\mathrm{al}^{49}$} & & $\begin{array}{l}\text { Exemestane } \\
(n=248)\end{array}$ & $\begin{array}{l}\text { Letrozole } \\
(\mathrm{n}=252)\end{array}$ & $P$ value \\
\hline & $\begin{array}{l}\text { Treatment } \\
\text { discontinuation due } \\
\text { to toxicity, } \%\end{array}$ & 36.7 & 28.6 & 0.02 \\
\hline & $\begin{array}{l}\text { Treatment } \\
\text { discontinuation for } \\
\text { reasons other than } \\
\text { toxicity, \% }\end{array}$ & 10.5 & 6.7 & \\
\hline & $\begin{array}{l}\text { Treatment } \\
\text { discontinuation due } \\
\text { to musculoskeletal } \\
\text { symptoms, \% }\end{array}$ & 26.6 & 22.2 & \\
\hline & $\begin{array}{l}\text { Median time } \\
\text { to treatment } \\
\text { discontinuation, } \\
\text { months }\end{array}$ & $\begin{array}{l}5.8 \text { (range } \\
0.2-20.3 \text { ) }\end{array}$ & $\begin{array}{l}8.1 \text { (range } \\
0.1-21.2 \text { ) }\end{array}$ & \\
\hline
\end{tabular}

suggests that nonadherence could affect a significant number of patients and has been associated with both extremes of age. Clearly more qualitative research is required to help identify individuals most at risk of noncompliance, in whom closer supervision in both primary and tertiary care may improve outcomes.

\section{Conclusion}

Current licensing by the Medicines and Healthcare products Regulatory Agency (MHRA) in the UK approves the use of exemestane in postmenopausal (natural or induced) ER-positive women in two settings, ie, as part of adjuvant sequential therapy, following 2-3 years of tamoxifen, to make 5 years in total, and in metastatic breast cancer, following failure on antiestrogen therapy.

In addition to and alongside these indications, key trials of exemestane in breast cancer in a number of settings in both early and advanced breast cancer have shown Phase III evidence supporting its efficacy (with some promising results in chemoprevention), but at the expense of toxicity, predominantly affecting bone, musculoskeletal, and cardiovascular health. This emphasizes the importance of patient-centered care. When deciding upon a treatment for a patient, their past medical history, quality of life, and psychosocial needs must be addressed in turn. Of utmost importance is the involvement of the patient in the decisionmaking process, because there are pros and cons with any treatment being considered.

\section{Disclosure}

The authors report no conflicts of interest in this work.

\section{References}

1. Kaufmann M, Bajetta E, Dirix LY, et al. Exemestane is superior to megestrol acetate after tamoxifen failure in postmenopausal women with advanced breast cancer: results of a phase III randomized double-blind trial. The Exemestane Study Group. J Clin Oncol. 2000;18(7):1399-1411.

2. Falandry C, Debled M, Bachelot T, et al. Celecoxib and exemestane verus placebo and exemestane in postmenopausal metastatic breast cancer patients: a double-blind phase III GINECO study. Breast Cancer Res Treat. 2009;116(3):501-508.

3. Dirix LY, Ignacio J, Nag S, et al. Treatment of advanced hormone-sensitive breast cancer in postmenopausal women with exemestane alone or in combination with celecoxib. J Clin Oncol. 2008;26(8): 1253-1259.

4. Bresalier RS, Sandler RS, Quan H, et al. Cardiovascular events associated with rofecoxib in a colorectal adenoma chemoprevention trial. $N$ Engl J Med. 2005;352(1):1092-1102.

5. Chia S, Gradishar W, Mauriac L, et al. Double-blind, randomized placebo controlled trial of fulvestrant compared with exemestane after prior nonsteroidal aromatase inhibitor therapy in postmenopausal women with hormone receptor-positive, advanced breast cancer: results from EFECT. J Clin Oncol. 2008;26(10):1664-1670. 
6. Mauriac L, Romieu G, Bines J. Activity of fulvestrant versus exemestane in advanced breast cancer patients with or without visceral metastases: data from the EFECT trial. Breast Cancer Res Treat. 2009;117(1):69-75.

7. Baselga J, Campone M, Piccart M, et al. Everolimus in postmenopausal hormone-receptor-positive advanced breast cancer. $N$ Engl J Med. 2012;366(6):520-529.

8. Paridaens RJ, Dirix L, Beex L, et al. Promising results with exemestane in the first-line treatment of metastatic breast cancer: a randomized phase II EORTC trial with a tamoxifen control. Clin Breast Cancer. 2000;1 Suppl 1:S19-S21.

9. Paridaens R, Dirix L, Lohrisch C, et al. Mature results of a randomized phase II multicenter study of exemestane versus tamoxifen as first-line hormone therapy for postmenopausal women with metastatic breast cancer. Ann Oncol. 2003;14(9):1391-1398.

10. Paridaens RJ, Dirix LJ, Beex LV, et al. Phase III study comparing exemestane with tamoxifen as first-line hormonal treatment of metastatic breast cancer in postmenopausal women: the European Organization for Research and Treatment of Cancer Breast Cancer Cooperative Group. J Clin Oncol. 2008;26(30):4883-4890.

11. Llombart-Cussac A, Ruiz A, Anton A, et al. Exemestane versus anastrozole as front-line endocrine therapy in postmenopausal patients with hormone receptor-positive, advanced breast cancer: final results from the Spanish Breast Cancer Group 2001-2003 phase 2 randomized trial. Cancer. 2012;118(1):241-247.

12. Van de Velde CJ, Rea D, Seynaeve C, et al. Adjuvant tamoxifen and exemestane in early breast cancer (TEAM): a randomized phase 3 trial. Lancet. 2011;377(9762):321-331.

13. Dowsett M, Cuzick J, Wale C, et al. Retrospective analysis of time to recurrence in the ATAC trial according to hormone receptor status: an hypothesis-generating study. J Clin Oncol. 2005;23(30):7512-7517.

14. Bartlett JMS, Brookes CL, Robson T, et al. Estrogen receptor and progesterone receptor as predictive biomarkers of response to endocrine therapy: a prospectively powered pathology study in the tamoxifen and exemestane adjuvant multinational trial. J Clin Oncol. 2011;29(12):1531-1538.

15. Coombes RC, Hall E, Gibson LJ, et al. A randomized trial of exemestane after two to three years of tamoxifen therapy in postmenopausal women with primary breast cancer. $N$ Engl J Med. 2004;350(11):1081-1092.

16. Coombes RC, Kilburn LS, Snowdon CF, et al. Survival and safety of exemestane versus tamoxifen after 2-3 years' tamoxifen treatment (Intergroup Exemestane Study): a randomised controlled trial. Lancet. 2007;369(9561):559-570.

17. Bliss JM, Kilburn LS, Coleman RE, et al. Disease-related outcomes with long-term follow-up: an updated analysis of the Intergroup Exemestane Study. J Clin Oncol. 2012;30(7):709-717.

18. Mamounas EP, Jeong JH, Wickerham DL, et al. Benefit from exemestane as extended adjuvant therapy after 5 years of adjuvant tamoxifen: intention-to-treat analysis of the National Surgical Adjuvant Breast and Bowel Project B-33 trials. J Clin Oncol. 2008;26(12):1965-1971.

19. Davies C, Pan H, Godwin J, et al. Long-term effects of continuing adjuvant tamoxifen to 10 years versus stopping at 5 years after diagnosis of oestrogen receptor-positive breast cancer: ATLAS, a randomized trial. Lancet. 2012;pii: S0140-6736(12)61963-61961.

20. Early Breast Cancer Trialists' Collaborative Group (EBCTCG). Effects of chemotherapy and hormonal therapy for early breast cancer on recurrence and 15 year survival: an overview of the randomized trials. Lancet. 2005;365(1):1687-1717.

21. Goss PE, Ingle JN, Martino S, et al. Randomized trial of letrozole following tamoxifen as extended adjuvant therapy in receptor-positive breast cancer: updated findings from NCIC CTG MA.17. J Natl Cancer Inst. 2005;97(17):1262-1271.

22. Ellis MJ, Suman VJ, Hoog J, et al. Randomized phase II neoadjuvant comparison between letrozole, anastrozole and exemestane for postmenopausal women with estrogen receptor-rich stage 2 to 3 breast cancer: clinical and biomarker outcomes and predictive value of the baseline PAM50-based intrinsic subtype - ACOSOG Z1031. J Clin Oncol. 2011;29(17):2342-2349.
23. Fisher B, Brown A, Mamounas E, et al. Effect of preoperative chemotherapy on local-regional disease in women with operable breast cancer: findings from National Surgical Adjuvant Breast and Bowel Project B-18. J Clin Oncol. 1997;15(7):2483-2493.

24. Chow LW, Yip AY, Loo WT, Lam CK, Toi M. Celecoxib anti-aromatase neoadjuvant (CAAN) trial for locally advanced breast cancer. J Steroid Biochem Mol Biol. 2008;111(1-2):13-17.

25. Cuzick J, Powles T, Veronesi U, et al. Overview of the main outcomes in breast-cancer prevention trials. Lancet. 2003;361(9354):296-300.

26. Goss PE, Ingle JN, Ales-Martinez JE, et al. Exemestane for breast-cancer prevention in postmenopausal women. $N$ Engl J Med. 2011;364(25): 2381-2391.

27. Atalay G, Dirix L, Biganzoli L, et al. The effect of exemestane on serum lipid profile in postmenopausal women with metastatic breast cancer: a companion study to EORTC Trial 10951, 'Randomized phase II study in first line hormonal treatment for metastatic breast cancer with exemestane or tamoxifen in postmenopausal patients.' Ann Oncol. 2004;15(2):211-217.

28. Fallowfield LJ, Bliss JM, Porter LS, et al. Quality of life in the intergroup exemestane study: a randomized trial of exemestane versus continued tamoxifen after 2 to 3 years of tamoxifen in postmenopausal women with primary breast cancer. J Clin Oncol. 2006;24(6):910-917.

29. Fallowfield LJ, Kilburn LS, Langridge C, et al. Long-term assessment of quality of life in the Intergroup Exemestane Study: 5 years postrandomisation. Br J Cancer. 2012;106(6):1062-1067.

30. Coleman RE, Banks LM, Girgis SI, et al. Skeletal effects of exemestane on bone-mineral density, bone biomarkers and fracture incidence in postmenopausal women with early breast cancer participating in the Intergroup Exemestane Study (IES): a randomized controlled study. Lancet Oncol. 2007;8(2):119-127.

31. Gonnelli S, Cadirni A, Caffarelli C, et al. Changes in bone turnover and in bone mass in women with breast cancer switched from tamoxifen to exemestane. Bone. 2007;40(1):205-210.

32. Geisler J, Lonning PE. Krag LE, et al. Changes in bone and lipid metabolism in postmenopausal women with early breast cancer after terminating 2-year treatment with exemestane: a randomised, placebocontrolled study. Eur J Cancer. 2006;42(17):2968-2975.

33. Bertelli G, Hall E, Ireland E, et al. Long-term endometrial effects in postmenopausal women with early breast cancer participating in the Intergroup Exemestane Study (IES) - a randomized controlled trial of exemestane versus continued tamoxifen after $2-3$ years tamoxifen. Ann Oncol. 2010;21(3):498-505.

34. Mieog JS, Morden JP, Bliss JM, Coombes RC, van de Velde CJ. Carpal tunnel syndrome and musculoskeletal symptoms in postmenopausal women with early breast cancer treated with exemestane or tamoxifen after 2-3 years of tamoxifen: a retrospective analysis of the Intergroup Exemestane Study. Lancet Oncol. 2012;13(4):420-432.

35. Jones SE, Cantrell J, Vukelja S, et al. Comparison of menopausal symptoms during the first year of adjuvant therapy with either exemestane or tamoxifen in early breast cancer: report of a Tamoxifen Exemestane Adjuvant Multicener trial substudy. J Clin Oncol. 2007;25(30): 4765-4767.

36. Kieback DG, Harbeck N, Bauer W, et al. Endometrial effects of exemestane compared to tamoxfien within the Tamoxifen Exemestane Adjuvant Multicenter (TEAM) trial: results of a prospective gynecological ultrasound substudy. Gynecol Oncol. 2010;119(3):500-505.

37. Markopoulos C, Polychronis A, Dafni U, et al. Lipid changes in breast cancer patients on exemestane treatment: final results of the TEAM Greek sub-study. Ann Oncol. 2009;20(1):49-55.

38. Markopoulos C, Dafni U, Misitzis, et al. Extended adjuvant hormonal therapy with exemestane has no detrimental effect on the lipid profile of postmenopausal breast cancer patients: final results of the ATENA lipid substudy. Breast Cancer Res. 2009;11(3):R35.

39. Hozumi Y, Suemasu K, Takei H, et al. The effect of exemestane, anastrazole and tamoxifen on lipid profiles in Japanese postmenopausal early breast cancer patients: final results of National Surgical Adjuvant Study BC 04, the TEAM Japan sub-study. Ann Oncol. 2011;22(8): 1777-1782. 
40. Hadji P, Ziller M, Kieback DG, et al. Effects of exemestane and tamoxifen on bone health within the Tamoxifen Exemestane Adjuvant Multicenter (TEAM) trial: results of a German 12-month, prospective, randomized substudy. Ann Oncol. 2009;20(7):1203-1209.

41. Hadji P, Ziller M, Kieback DG, et al. The effect of exemestane or tamoxifen on markers of bone turnover: results of a German sub-study of the Tamoxifen Exemestane Adjuvant Multicentre (TEAM) trial. Breast. 2009;18(3):159-164.

42. Schilder CM, Eggens PC, Seynaeve C, et al. Neuropsychological functioning in postmenopausal breast cancer patients treated with tamoxifen or exemestane after AC-chemotherapy: cross-sectional findings from the neuropsychological TEAM side-study. Acta Oncol. 2009;48(1):76-85.

43. Schilder CM, Seynaeve C, Beex LV, et al. Effects of tamoxifen and exemestane on cognitive functioning of postmenopausal patients with breast cancer: results from the neuropsychological side study of the tamoxifen and exemestane adjuvant multinational trial. J Clin Oncol. 2010;28(8):1294-1300.

44. Goss PE, Hadji P, Subar M, et al. Effects of steroidal and nonsteroidal aromatase inhibitors on markers of bone turnover in healthy postmenopausal women. Breast Cancer Res. 2007;9(4):R52.

45. Cigler T, Richardson H, Yaffe MJ, et al. A randomized, placebocontrolled trial (NCIC CTG MAP.2) examining the effects of exemestane on mammographic breast density, bone density, markers of bone metabolism and serum lipid levels in postmenopausal women. Breast Cancer Res Treat. 2011;126(2):453-461.
46. Cheung AM, Tile L, Cardew S, et al. Bone density and structure in healthy postmenopausal women treated with exemestane for the primary prevention of breast cancer: a nested substudy of the MAP.3 randomised controlled trial. Lancet Oncol. 2012;13(3):275-284.

47. Reid DM, Doughty J, Eastell R, et al. Guidance for the management of breast cancer treatment-induced bone loss: a consensus position statement from a UK expert group. Cancer Treat Rev. 2008;34 Suppl 1:S3.

48. Sedjo RL, Devine S. Predictors of non-adherence to aromatase inhibitors among commercially insured women with breast cancer. Breast Cancer Res Treat. 2011;125(1):191-200.

49. Henry NL, Desta Li L, Nguyen AT, et al. Predictors of aromatase inhibitor discontinuation as a result of treatment-emergent symptoms in early-stage breast cancer. J Clin Oncol. 2012;30(9): 936-942.

50. Van de Water W, Bastiaannet E, Hille ET, et al. Age-specific nonpersistence of endocrine therapy in postmenopausal patients diagnosed with hormone receptor-positive breast cancer: a TEAM study analysis. Oncologist. 2012;17(1):55-63.
Patient Preference and Adherence

\section{Publish your work in this journal}

Patient Preference and Adherence is an international, peer-reviewed, open access journal focusing on the growing importance of patient preference and adherence throughout the therapeutic continuum. Patient satisfaction, acceptability, quality of life, compliance, persistence and their role in developing new therapeutic modalities and compounds to

\section{Dovepress}

optimize clinical outcomes for existing disease states are major areas of interest. This journal has been accepted for indexing on PubMed Central. The manuscript management system is completely online and includes a very quick and fair peer-review system. Visit http://www.dovepress.com/ testimonials.php to read real quotes from published authors. 